\title{
BOLD Variability is Related to Dopaminergic Neurotransmission and Cognitive Aging
}

\section{Marc Guitart-Masip ${ }^{1,2}$, Alireza Salami1,3, Douglas Garrett ${ }^{4}$, Anna Rieckmann Ulman Lindenberger ${ }^{4}$, and Lars Bäckman ${ }^{1}$}

\author{
${ }^{1}$ Aging Research Center, Karolinska Institute, SE-113 30 Stockholm, Sweden, ${ }^{2}$ Wellcome Trust Center for \\ Neuroimaging, University College London, WC1N 3BG London, UK, ${ }^{3}$ Umeå Center for Functional Brain Imaging, \\ S-90187 Umeå, Sweden, ${ }^{4}$ Max Plank Institute for Human Development, 14195 Berlin, Germany, and ${ }^{5}$ Center for \\ Brain Science, Harvard University, Cambridge, MA 02138, USA
}

Address correspondence to Marc Guitart-Masip. Email: marc.guitart-masip@ki.se

\begin{abstract}
Dopamine (DA) losses are associated with various aging-related cognitive deficits. Typically, higher moment-to-moment brain signal variability in large-scale patterns of voxels in neocortical regions is linked to better cognitive performance and younger adult age, yet the physiological mechanisms regulating brain signal variability are unknown. We explored the relationship among adult age, DA availability, and blood oxygen level-dependent (BOLD) signal variability, while younger and older participants performed a spatial working memory (SWM) task. We quantified striatal and extrastriatal DA D1 receptor density with $\left[{ }^{11} \mathrm{C}\right] \mathrm{SCH} 23390$ and positron emission tomography in all participants. We found that BOLD variability in a neocortical region was negatively related to age and positively related to SWM performance. In contrast, BOLD variability in subcortical regions and bilateral hippocampus was positively related to age and slower responses, and negatively related to D1 density in caudate and dorsolateral prefrontal cortex. Furthermore, BOLD variability in neocortical regions was positively associated with task-related disengagement of the default-mode network, a network whose activation needs to be suppressed for efficient SWM processing. Our results show that age-related DA losses contribute to changes in brain signal variability in subcortical regions and suggest a potential mechanism, by which neocortical BOLD variability supports cognitive performance.
\end{abstract}

Key words: BOLD variability, cognitive aging, default mode network, dopamine, spatial working memory

\section{Introduction}

Dopaminergic neurotransmission has a well-established role in supporting cognitive functions, including working memory (Cools and D'Esposito 2011), episodic memory (Frey and Morris 1997; Lisman and Grace 2005), invigoration of motivated behavior (Niv et al. 2007; Salamone et al. 2007; Beierholm et al. 2013), and reward learning (Schultz et al. 1997). Gradual losses of dopamine (DA) functions across the adult life span have been linked to cognitive deficits in aging (for reviews, see Backman et al. 2006, 2010). Indeed, recent pharmacological studies have demonstrated a direct association between DA activity and cognitive performance (Fischer et al. 2010; Chowdhury et al. 2013). In these studies, the link between DA levels and neural processing in brain regions supporting specific cognitive functions referred to measures of central tendency [e.g., blood oxygen-level-dependent (BOLD) mean]. However, recent studies have demonstrated that measures of variability in neural responses (e.g., BOLD standard deviation) provide complementary information when characterizing the effects of aging on brain function (for review see Garrett, Samanez-Larkin et al. 2013). These studies have shown that BOLD variability in neocortical regions is reduced in older adults (Garrett et al. 2010, 2011; Garrett, Kovacevic et al. 2013), and generally supports cognitive performance across different tasks (Garrett et al. 2011; Garrett, Kovacevic et al. 2013). Only in some 
restricted regions (e.g., striatum, hippocampus) BOLD variability is higher in older adults and relates to poorer cognitive performance (Garrett et al. 2010, 2011; Samanez-Larkin et al. 2010).

Thus, overall brain signal variability across large-scale brain regions has emerged as a marker of a well-functioning brain (Faisal et al. 2008; McIntosh et al. 2008, 2010; Garrett et al. 2010; 2011; Garrett, Kovacevic et al. 2013; Garrett, McIntosh et al. 2014; Garrett, Samanez-Larkin et al. 2013). According to stochastic resonance theory, an optimal level of neural variability facilitates detection of weak incoming signals (Li et al. 2001; McDonnell and Ward 2011). Signal variability can also reflect greater dynamic range, which is generally beneficial to the adaptability and efficiency of neural systems, as it permits a greater range of responses to a greater range of stimuli (McIntosh et al. 2010; Deco et al. 2011; Garrett, Samanez-Larkin et al. 2013). However, despite the increasingly recognized functional importance of BOLD variability, little is known about its neural underpinnings, and the origins of its regionally dependent changes associated with aging. The dopaminergic system is a good candidate to affect levels of BOLD variability, given well-established links between DA signaling and BOLD activity (Landau et al. 2009; Nyberg et al. 2009; Backman, Nyberg et al. 2011; GuitartMasip et al. 2012).

We examined for the first time the relationship of BOLD variability to aging, DA functions, and cognitive performance. In doing so, we analyzed a dataset involving positron emission tomography (PET) measures of D1 receptor density in younger (22-30 years old) and older (65-75 years old) adults, and functional magnetic resonance imaging (fMRI) data acquired while the same participants performed a spatial working memory (SWM) task under 2 load conditions (Fischer et al. 2010). We used partial least squares analysis (McIntosh et al. 1996; Krishnan et al. 2011) to identify multivariate patterns of brain activity that covary with age, cognitive performance, and D1 receptor density (McIntosh et al. 1996; Krishnan et al. 2011). We expected lower neocortical and higher subcortical BOLD variability in old age (Garrett et al. 2010, 2011; Garrett, Kovacevic et al. 2013). If BOLD variability in cortical and subcortical regions is affected similarly by lower DA levels, our PLS analysis should reveal a single pattern. Alternatively, if lower DA levels have a differential impact on BOLD variability in cortical and subcortical regions, we would expect 2 distinct patterns in the PLS analysis. Furthermore, we explored whether age differences in BOLD variability in cortical and subcortical regions predicted task-related activation or task-related deactivation of broad patterns of voxels in the brain, and whether one of the 2 or both may be regarded as a candidate mechanism underlying age-related cognitive decline.

\section{Materials and Methods}

\section{Participants}

Participants were 19 healthy younger adults ( 9 women; mean age $=25.16, \mathrm{SD}=2.27$ ) and 18 older adults ( 9 women; mean age $=$ $70.33, \mathrm{SD}=3.25$ ). They were recruited by newspaper advert. There was no difference in years of education between age groups (mean younger $=14.61, \mathrm{SD}=2.00$; mean older $=14.72, \mathrm{SD}=3.96$; $t>1$ ), and all participants were right handed, nonsmokers, and free from past or present drug or alcohol abuse, any neuropsychiatric disorders (including mood disorders, schizophrenia, movement disorders, or dementia), or brain damage. No specific dementia screening tools (e.g., MMSE) were administered as part of study enrollment. However, as previously published (MacDonald et al. 2012), cognitive testing outside the PET system revealed that the 2 samples were highly representative of their birth cohorts: There was a clear advantage of the young in a test of episodic memory (Free recall of words: mean young $=11.78$ $[\mathrm{SD}=2.39]$, mean old $=9.60$ [SD $=2.46], t=2.76, P<0.01$ ). In contrast, the old outperformed the young in tests of crystallized intelligence (Vocabulary: mean young $=29.39$ [ $S D=2.62$ ], mean old $=33.30$ [SD=2.00], $t=-5.21, P<0.01$; General knowledge: mean young $=23.06[\mathrm{SD}=2.31]$, mean old $=25.30$ [SD $=3.08], t=$ $-2.52, P=0.02$ ). This pattern of data makes it highly unlikely that any of the older participants suffered from dementia. Furthermore, the same episodic memory test was administered to a large group of subjects in an ongoing population-based study of older persons at our research center. Data from same-aged subjects from the SNAC-K population-based study (Free recall of words: mean $60-66$ years old $=7.96$ [SD $=2.49$ ]; Vocabulary: mean 60-66 years old $=24.06[\mathrm{SD}=4.51]$; General knowledge mean 60-66 years old $=7.12$ [SD = 1.52] Laukka et al. 2013) underscore the point that the current old subjects were normally functioning cognitively. Written informed consent was obtained from all participants, and the Ethics and Radiation Safety Committees of the Karolinska Hospital, Stockholm, Sweden, approved the study.

\section{Procedure}

All participants took part in PET and fMRI assessments not separated by more than 2 months. The PET and fMRI sessions lasted $\sim 1 \mathrm{~h}$ each. During the PET measurement, participants were instructed to rest. During fMRI acquisition, participants performed a SWM task.

\section{Working Memory Task}

During fMRI acquisition, participants performed a SWM task, which has been described in detail previously (Fischer et al. 2010; Backman, Karlsson et al. 2011; Rieckmann et al. 2011). Briefly, participants were presented with a $4 \times 4$ grid at the center of a computer screen. For each SWM trial, either 4 (low load) or 6 (high load) target circles consecutively lit up in random positions of the grid for $900 \mathrm{~ms}$ each circle. After a delay of $1500 \mathrm{~ms}$, a probe circle lit up and participants were to indicate by button press whether any of the target circles had appeared in the position of the probe circle. In total, participants were presented with 15 high-load and 15 low-load trials presented in blocks (3 trials per block). Accuracy and mean reaction time (RT) for correct responses were computed for each participant and load condition and used in our behavioral PLS analysis (see below).

A perceptually matched baseline condition, referred here as control condition, was alternated with SWM blocks. In the control condition, participants were also instructed to watch 4 or 6 green filled circles that were presented one-by-one in each of the 4 corners of the grid. After a 1500-msec delay, an unfilled green circle (probe) appeared in the middle of the grid, and participants responded by pressing any button with their index finger each time the probe appeared.

\section{PET data Acquisition and Analysis}

Dynamic PET data were obtained with an ECAT Exact HR 47 system (CTI/Siemens) run in 3D mode and with a transaxial resolution of $3.8 \mathrm{~mm}$ FWHM at the central field of view and $4.5 \mathrm{~mm}$ radially at $20 \mathrm{~cm}$ from the center. Emission data were recorded over a period of $51 \mathrm{~min}$ in 13 time frames after a transmission measurement of $10 \mathrm{~min}$ with 3 rotating ${ }^{68} \mathrm{Ge} /{ }^{68} \mathrm{Ga}$ sources. 
${ }^{[11 C]}$ SCH23390 (300 MBq) (Halldin et al. 1986) was injected into the left antecubital vein. A plaster helmet was made for each participant individually and used to fixate the head and minimize motion. Light was dimmed and auditory stimulation was kept to a minimum during acquisition of the PET data.

All PET analyses were performed using SPM5 (http://www.fil. ion.ucl.ac.uk/spm/) and in-house scripts implemented through MATLAB R2007b. Bilateral caudate, DLPFC, and cerebellum were manually delineated on the structural MRI scans for each individual using the Human Brain Atlas software (Roland et al. 1994). DLPFC was delineated as the medial-inferior and lateral part of superior frontal gyrus. The cerebellum, where DA D1 receptor density is negligible, was delineated on 6 central slices and served as reference region (Hall et al. 1988). After manual delieation, MRI images were coregistered with the PET images and segmented into gray matter (GM), white matter (WM), and CSF using SPM's mutual information coregistration. The manually delineated ROIS were masked with the GM segmentation and used to derive regional time activity curves. This approach minimizes partial-volume effects (inclusion of WM and CSF in the PET signal) in manually delineated ROIs. An example of this procedure is described and shown in MacDonald et al. (2012). Regional radioactivity was calculated for each frame, corrected for decay, and plotted versus time. D1 receptor density was measured as the binding potential (BP) of ${ }^{[11 C]} \mathrm{SCH} 23390$, pooled across hemispheres. Here, BP is the ratio at equilibrium of specifically bound radioligand to that of nondisplaceable radioligand in tissue (Innis et al. 2007) and calculated using the simplified reference tissue model, with cerebellum GM as reference region (Hall et al. 1988). The PET data were corrected for partial-volume effects (Meltzer et al. 1990).

To constrain the number of solutions of the PLS analysis and facilitate the interpretation of PLS patterns, PET measures used in the analysis were restricted to caudate and DLPFC. DA function in caudate has a recognized role in WM performance and WMrelated fMRI activations (Landau et al. 2009; Backman et al. 2011), and DLPFC is one of the key cortical regions supporting WM performance (Curtis and D'Esposito 2003). Although DA transmission in other regions may be related to BOLD variability, D1 receptor densities are highly correlated across regions in this dataset (Rieckmann et al. 2011). Thus, by selecting caudate and DLPFC, we are likely to sample enough regional variability of D1 density to be able to examine the link between dopaminergic neuromodulation and BOLD variability.

\section{MRI data Acquisition and Preprocessing}

All MR images were acquired on a $1.5 \mathrm{~T}$ system (Signa Excite HD Twinspeed, General Electrics Medical Systems). Participants viewed stimuli from a projector (Philips Hopper HG 20 Impact LCD projector) via a coil mirror, and responded via an fMRIcompatible button set using the right index and middle finger (Psychology Software Tools). A $\mathrm{T}_{1}$-weighted 3D-SPGR sequence (time repetition $[\mathrm{TR}]=24 \mathrm{~ms}$, time echo $[\mathrm{TE}]=6 \mathrm{~ms}$, flip angle $=35^{\circ}$, slice thickness $1.5 \mathrm{~mm}$, field of view [FOV] $256 \mathrm{~mm}$ ) was used for coregistration with the functional MRI and PET images. Functional data were collected using an EPI sequence $(T R=2.5 \mathrm{~s}, \mathrm{TE}=40 \mathrm{~ms}$, flip angle $=90^{\circ}$, slice thickness $=4 \mathrm{~mm}$ with $0.5 \mathrm{~mm}$ gap, interleaved, FOV $220 \mathrm{~mm}$ ). Two EPI runs of 140 volumes each were entered into the fMRI analyses, after removal of the first 4 volumes as dummy scans.

For the normalization process, a rigid within-subject registration was conducted to align functional and structural images together. $\mathrm{T}_{1}$-weighted images were segmented into GM and WM using a new segmentation algorithm implemented in SPM8 [modified version of unified segmentation by (Ashburner and Friston 2005)]. Then, a group-specific template was created using Diffeomorphic Anatomical Registration using Exponentiated Lie Algebra (DARTEL) (Ashburner 2007). This was done by first importing tissue class images (e.g., GM, WM) into the DARTEL space using the normalization parameter yielded during the segmentation step followed by resampling to isotropic voxels $\left(1.5 \_1.5 \_1.5 \mathrm{~mm}\right)$. Then, the imported images went through an iterative procedure that began by producing an initial template as a mean of GM/WM across all participants $(n=37)$. Deformation from the initial template to each of the subject-specific GM/WM images was computed and the inverse of the deformation was applied to each of the subject-specific GM/WM images. A second template was then created as the mean of the deformed subjectspecific GM/WM images across all participants and this procedure was repeated until a sixth template was created. Finally, the coregistered fMRI images and segmented GM/WM images were nonlinearly normalized, subject by subject, to the sample-specific template (using a subject-specific flow field), affine aligned into a MNI template, and smoothed using a $8.0 \mathrm{~mm}$ full-width at halfmaximum Gaussian filter. Thus, both preprocessed structural MRI and fMRI images were in the same space and had the same voxel size $(1.5 \times 1.5 \times 1.5 \mathrm{~mm})$.

To reduce data artifacts, we corrected functional volumes in the normalized space via independent component analysis with separate runs using GIFTv2.0a (Medial Image Analysis Lab). Components including spikes, motion artifacts, susceptibility and flow artifacts, signal in ventricles or WM, low frequency signal drifts, high power in high frequencies, and spotty pattern distribution were judged to be potential artifacts. The selection was based on criteria previously used as a preprocessing benchmark in studies of BOLD variability (Garrett et al. 2010, 2011; Garrett, Kovacevic et al. 2013; Garrett, McIntosh et al. 2014). Selected artifact components were regressed from each voxel's time series, and the data were reconstructed.

\section{MRI data Analysis}

\section{Calculation of BOLD Signal Mean and SD}

The mean signal during high load, low load, and control blocks was calculated at each voxel. We expressed each measurement as a percentage signal change from its respective block onset (14 volumes/block), calculated a mean percentage change within each block, and finally averaged across blocks in the same condition. To calculate BOLD SDs during high load, low load, and control blocks, we performed an additional block normalization as previously described in studies of BOLD variability (Garrett et al. 2010, 2011; Garrett, Kovacevic et al. 2013; Garrett, McIntosh et al. 2014). In short, we first normalized all blocks such that the overall 4-dimensional mean across brain and blocks was 100. For each voxel, we then subtracted the block mean and concatenated across all condition-specific blocks. Finally, we calculated SDs for each voxel across this concatenated mean block-corrected time series.

\section{Behavioral Partial Least Squares (PLS) Analysis}

We used behavioral PLS analysis (McIntosh et al. 1996; Krishnan et al. 2011) to identify multivariate patterns of brain activity $\left(\mathrm{SD}_{\text {BOLD }}\right.$ or mean $\left.\mathrm{BOLD}_{\mathrm{B}}\right)$ that covary with the measures of interest; the so-called behavioral matrix included 5 variables: age, SWM accuracy, SWM RT, D1 BP in caudate, and D1 BP in DLPFC.

Behavioral PLS proceeded in several steps. First, a correlation matrix between the behavioral matrix and each voxel signal in 
the high- and low-load conditions were calculated across subjects. In the second stage, the correlation matrix was decomposed using single-value decomposition to produce latent variables. In the current study, 10 latent variables were obtained for each behavioral PLS analysis (5 behavioral variables $\times$ BOLD measures in the 2 load conditions). For each latent variable, we obtained a singular value that represents the variance explained by each latent variable, and brain saliences, which are a weighting pattern across brain voxels representing the strength of the correlation between BOLD and the behavioral variables. In a third step, we calculated brain scores by taking the dot product of the voxel saliences and a given subject's brain images (representing either mean $\mathrm{B}_{\mathrm{BOLD}}$ or $\mathrm{SD}_{\mathrm{BOLD}}$ in each load condition). This single measure reflects the degree to which a subject expresses the multivariate spatial pattern captured by a given set of measures (age, SWM accuracy and RT, and D1 BP in caudate and DLPFC).

The significance of each latent variable and the robustness of voxel saliences were assessed using 1000 permutation tests and 1000 bootstrapped resamples, respectively. By dividing each voxel's bootstrap mean salience by its estimated standard error, we obtained bootstrap ratios as normalized estimates of robustness. We thresholded bootstrapped ratios at a value of 2.97 [akin to a $z$-score of 2.97, corresponding approximately to $P<0.001$ ]. In addition, upper and lower percentiles of the bootstrap distribution were used to generate $95 \%$ confidence intervals (CIs) to facilitate interpretation of links between brain scores and behavioral variables. Relations were deemed unreliable when CIs crossed zero. Participants with brain scores more than 3 standard deviations from the mean were excluded from analysis and the PLS was recalculated. As a result, one older participant was excluded from the mean BOLD analysis, and one younger and 2 older participants were excluded from the BOLD variability analysis.

\section{Task Partial Least Squares (PLS) Analysis}

We used task PLS analysis (McIntosh et al. 1996; Krishnan et al. 2011) on the mean BOLD data to assess how brain activity is modulated across different task conditions and groups (younger participants, older participants) and derived summary scores of the task-related activation and/or deactivation of the detected pattern of voxels. This multivariate analysis assesses how brain activity is modulated across different conditions (high-load SWM, low-load SWM, control condition) and groups (younger participants; older participants) (McIntosh et al. 1996; Krishnan et al. 2011). In addition, this analysis uses all conditions in an experiment at once, and thus provides an additional dimension to data by simultaneously considering indices of both similarities and differences across groups and experimental variables.

Similar to behavioral PLS, task PLS is also performed in stages. In the first stage, the grand mean of each participant's BOLD signal was subtracted from each condition and a cross-covariance matrix between experimental conditions and each voxel signal was calculated across subjects for each group (younger participants; older participants) separately. The remaining stages of the analysis did not differ from the behavioral PLS (see above) and involved single-value decomposition and calculation of 3 brain scores per participant, one for each experimental condition. A significant difference between brain scores in different conditions is indicated by nonoverlapping bootstrapped CIs. Using our 3 standard deviations cutoff for inclusion as noted above, one younger participant under antagonist and one older participant were excluded from this analysis. To summarize the activation/deactivation patterns as identified by the task PLS, we computed a composite score such that the brain scores of the high- and low-load conditions from the task PLS analysis were averaged for each participant, and then subtracted from the brain score of the control condition.

\section{Detection of Possible Outliers: Cook's Distance}

We calculated Cook's Distance (Cook 1977, 1979) to detect possible outliers in the univariate analyses assessing the relationship between the switch scores (task PLS analysis) and the brain scores that summarize the relationship of $\mathrm{SD}_{\mathrm{BOLD}}$ to age, SWM accuracy, SWM RT, and D1 density in caudate and DLPFC (behavioral PLS analysis). The Cook's Distance reflects the extent to which model residuals would change if a particular subject's data (in multivariate space) were excluded from regression coefficient estimation. Larger Cook's Distance values indicate more influential subjects. Our threshold for determining influential observations was set according to previous recommendations [ $=4 /$ number of subjects $=4 / 37=0.11$ (Bollen and Jackman 1990) . Crucially, Cook's distance is agnostic to the direction of effect a participant may influence; thus, an outlying subject may drive either the over- or underestimation of "true" effects. Using this measure, we detected 3 potential outliers [Cook's distance $>0.2$ $(0.46,0.34,0.30$, highlighted in Fig. 3], whereas all other participants had a distance between 0 and 0.11 .

\section{Results}

\section{Relationships of $\mathrm{SD}_{\mathrm{BOLD}}$ to age, SWM Performance, and D1 Receptor Density}

We first used behavioral PLS (McIntosh et al. 1996; Krishnan et al. 2011) to assess whether there was any multivariate spatial pattern of $\mathrm{SD}_{\mathrm{BOLD}}$ dependent on age, SWM accuracy, SWM reaction time (RT), and D1 density in caudate and DLPFC (see methods). To constrain the number of solutions of the PLS analysis and facilitate the interpretation of PLS patterns, PET measures used in the analysis were restricted to bilateral caudate and DLPFC. DA function in caudate has a recognized role in WM performance and WM-related fMRI activations (Landau et al. 2009; Backman, Nyberg et al. 2011), and DLPFC is one of the key cortical regions supporting WM performance (Curtis and D'Esposito 2003).

We found 2 significant latent variables (LVs). LV1 $(P<0.05)$ accounted for $53.9 \%$ of the cross-block covariance (see methods for details), and uncovered a relationship of $\mathrm{SD}_{\mathrm{BO}}$ to age, $\mathrm{D} 1$ density in caudate and DLPFC, and RT in the SWM task. No reliable correlation was observed for accuracy for this LV (bootstrapped 95\% CIs crossed zero). The majority of regions contributing to this pattern of voxels ( $85.8 \%$ of robust voxels, in blue) was located in subcortical regions (amygdala, caudate, insula, hippocampus), and exhibited greater variability in older and slower adults, and in those with low D1 density in caudate and DLPFC for both load conditions (Fig. 1AC, see Supplementary Table 1). Conversely, a few voxels in posterior cingulate cortex and medial prefrontal cortex (in red) exhibited greater variability in younger and faster adults, and in those with higher D1 density in caudate and DLPFC (Fig. 1A,C, see Supplementary Table 1).

LV2 $(P<0.05)$ accounted for $29.97 \%$ of the cross-block covariance and revealed a relationship of $\mathrm{SD}_{\mathrm{BOLD}}$ to age and $\mathrm{SWM}$ accuracy for both load conditions (Fig. 1B). No reliable correlations were observed for RT and D1 density in caudate or DLPFC in either load condition (CIs crossed zero). Robust voxels (in yellow/red) were located in cortical regions (posterior cingulate cortex, medial prefrontal cortex, occipital cortex) and exhibited greater BOLD variability in younger and more accurate adults (Fig. $1 B, D$, see 
A
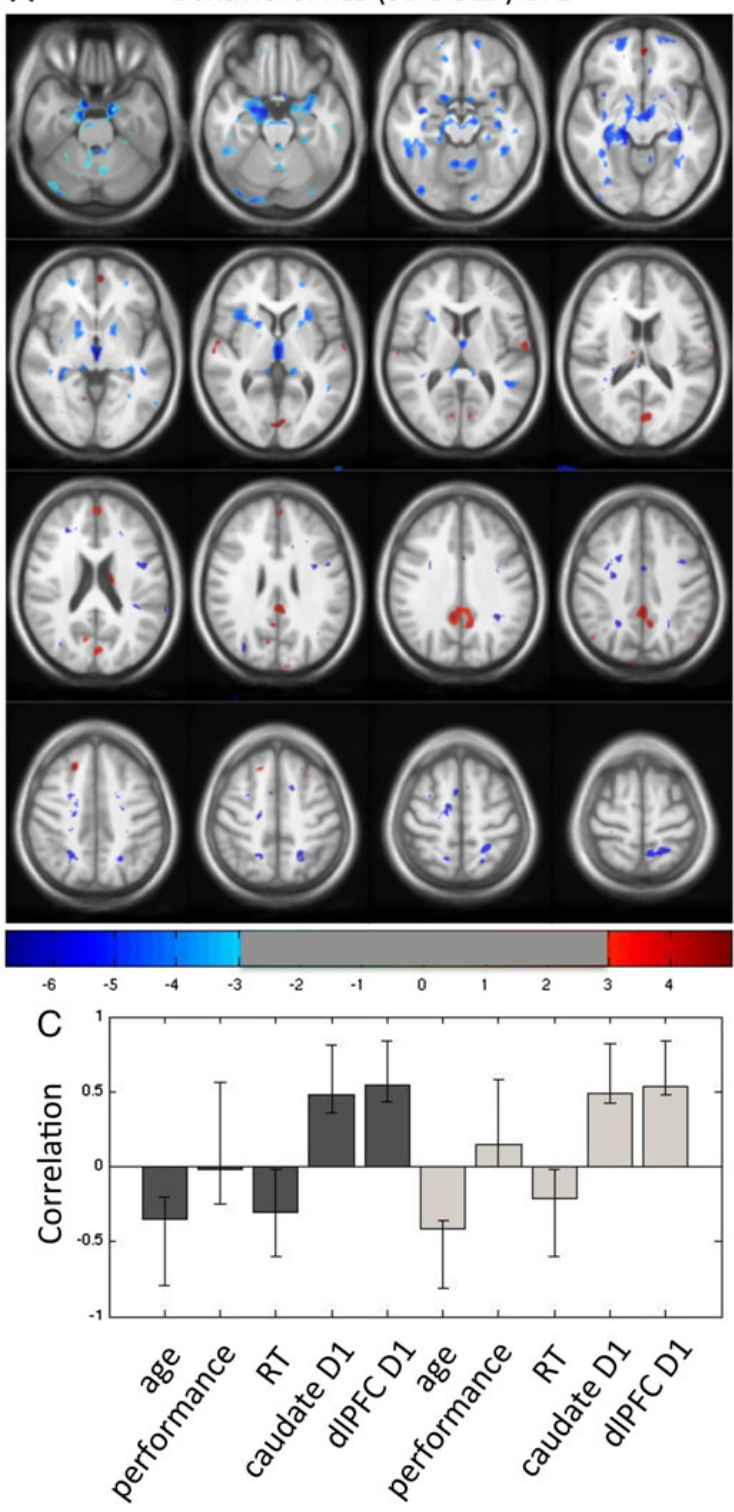

high load
B Behavioral PLS (SD BOLD) LV2
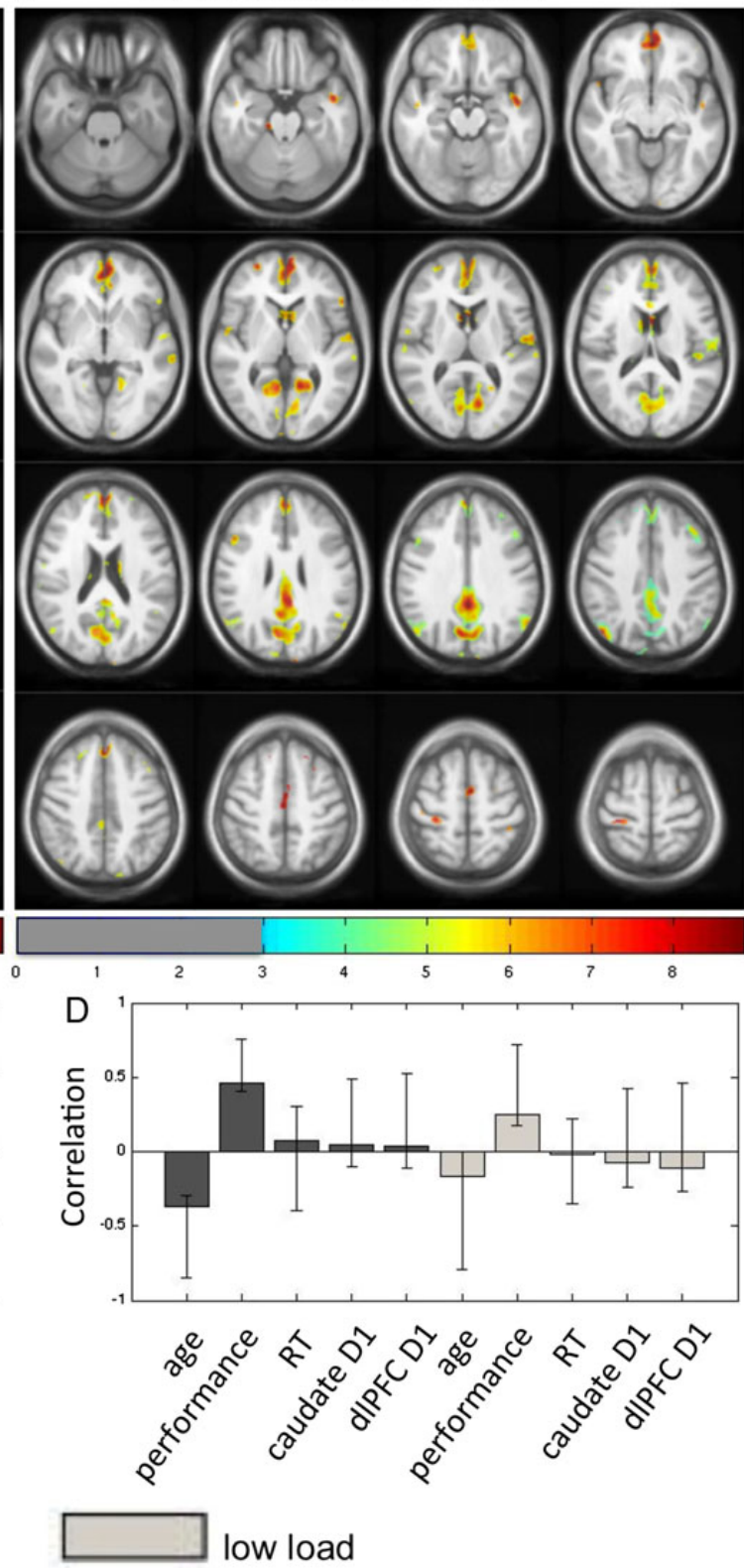

Figure 1. Multivariate relationships of BOLD variability to age, task performance, and D1 density. (A) Blue regions indicate higher and red regions reflect lower BOLD variability in older and slower adults with lower D1 density in caudate and DLPFC. The corresponding correlations are portrayed in (C). (B) All depicted regions indicate higher BOLD variability in younger adults with more accurate spatial working-memory (SWM) performance. The corresponding correlations are portrayed in (D). All robust areas in (A) and (B) surpassed a thresholded bootstrap ratio (salience/SE) of $>2.97$ for yellow and red regions and of less than -2.97 for blue regions. (C,D) Correlations (Pearson's $r$ ) of BOLD standard deviation during the low-load (light gray) and high-load (dark gray) conditions with age, SWM accuracy, SWM reaction time, D1 density in caudate, and D1 density in DLPFC. Error bars represent bootstrapped 95\% CIs.

Supplementary Table 2). No region with negative weights was observed for this LV.

Our behavioral PLS analysis unveiled 2 distinct patterns of voxels: one predominantly expressed in subcortical regions including the hippocampus, and the other predominantly expressed in neocortical regions. In subcortical regions, BOLD variability was higher with advancing age and lower DA density (LV1), whereas BOLD variability in neocortical regions was lower with advancing age (LV2). To rule out the possibility that these associations were driven by a confounding factor such as motion during the scanning session or brain atrophy, we conducted supplemental regression analyses (Supplementary Table 3). These analyses showed that the relationship between D1 receptor density and BOLD variability in subcortical regions was independent of head motion (during the fMRI acquisition) and gray-matter volumes. In addition, the relationship between age and BOLD variability in neocortical areas, depicted by LV2, persisted after controlling for D1 receptor density, head motion, and gray-matter volume.

\section{Relationship Between $\mathrm{SD}_{\mathrm{BOLD}}$ and Task-related mean $\mathrm{BOLD}_{\mathrm{BO}}$ Changes in Large Patterns of Voxels}

Older age was associated with lower $\mathrm{SD}_{\mathrm{BOLD}}$ in neocortical areas and higher $\mathrm{SD}_{\mathrm{BOLD}}$ in subcortical regions. In both cases, 
A

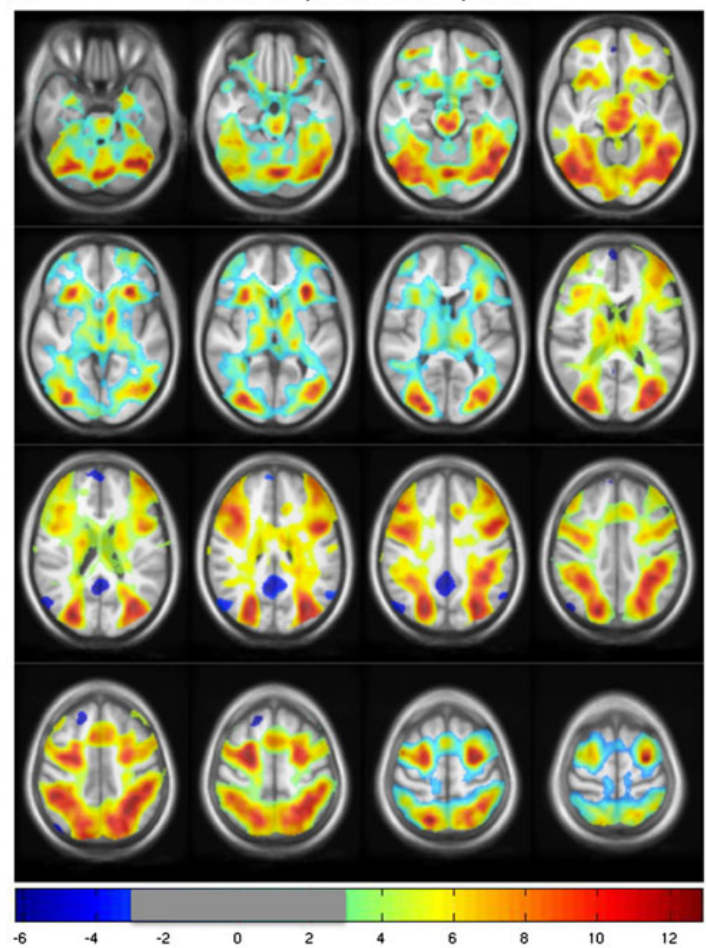

C

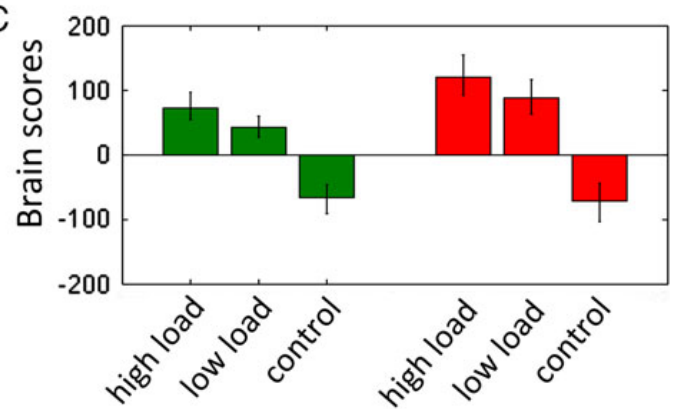

younger participants
B
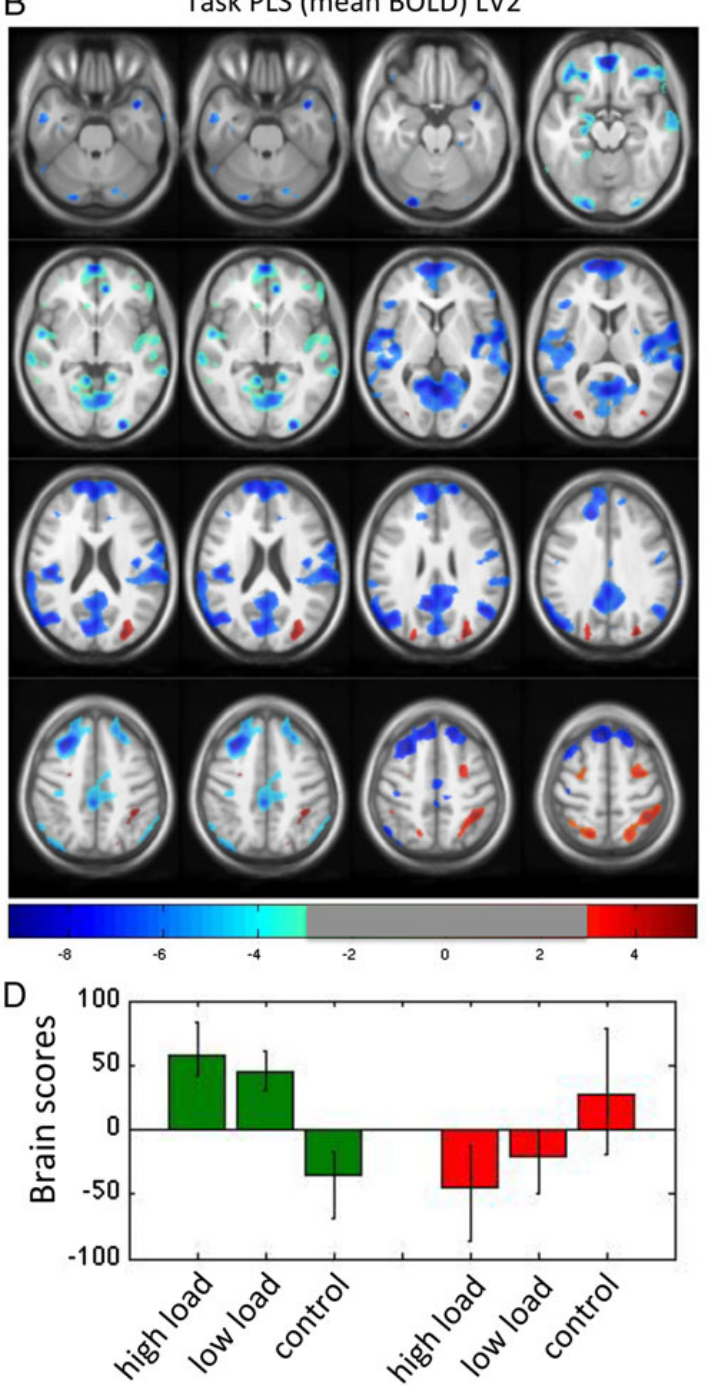

older participants

Figure 2. Age effects on the multivariate relationship between mean BOLD activity and task performance (A) Red, yellow, and green regions indicate higher and blue regions reflect lower BOLD mean during the 2 load conditions than in the control condition for both age groups. (B) Blue and green regions indicate lower and red regions reflect higher BOLD mean during the load conditions than the control condition in younger adults. In older adults, the pattern is reversed. The corresponding brain scores are depicted in (D). All robust areas in (A) and (B) surpassed a thresholded bootstrap ratio (salience/SE) of $>2.97$ for yellow and red regions and of less than -2.97 for blue regions. (C,D) Brain scores for each task condition (high load, low load, control) are plotted separately for each age group: younger adults (green) and older adults (red). Error bars represent bootstrapped $95 \%$ CIs.

age-related differences in $\mathrm{SD}_{\mathrm{BOLD}}$ were associated with poorer SWM performance. One possible mechanism by which agerelated differences in $\mathrm{SD}_{\mathrm{BOLD}}$ could be associated with poorer performance is by disrupting the dynamics of task-related activation and/or deactivation of broad patterns of voxels. To test this possibility we first used task PLS analysis (McIntosh et al. 1996; Krishnan et al. 2011) to detect task-related (mean BOLD $_{\text {) }}$ patterns of voxels that are similarly and/or differentially modulated across different conditions (control, low load, high load) and age groups. Thereafter, we computed a composite score summarizing the subject-specific strength of the task-related activation and/or deactivation of the detected patterns of voxels and assessed their association to the similar composite measure extracted from our initial behavioral PLS (i.e., brain scores summarizing age- and DA-dependent patterns of $\left.\mathrm{SD}_{\mathrm{BOLD}}\right)$.
In the task PLS analysis, we found 2 significant LVs. LV1 $(P<$ 0.001 ) accounted for $77 \%$ of the cross-block covariance and identified brain regions differentiating the 2 SWM conditions from the control condition. This pattern was similarly expressed in both age groups. Regions showing greater activity during both SWM conditions were located in cortical regions spanning a frontoparietal regions typically associated with working-memory processes (Fig. 2A (red color), C, see Supplementary Table 4) (Wager and Smith 2003), extending to other cortical and subcortical regions (e.g., occipital cortex, striatum, thalamus, midbrain). Brain regions showing relatively more activity during the control condition compared with the SWM conditions (Fig. 2A (blue color),2, see Supplementary Table 4) included posterior cingulate cortex, bilateral angular gyrus, medial frontal gyrus, bilateral middle temporal gyrus, and bilateral precuneus. These are 
canonical nodes of the default-mode network (DMN), which typically exhibits higher BOLD responses during rest and whose activity is suppressed when participants engage in an externally oriented cognitive task (Raichle et al. 2001; Buckner et al. 2008).

LV2 $(P<0.01$, Fig. 2, right panel) accounted for $18.9 \%$ of the cross-block covariance and also separated the 2 SWM load conditions from the control condition in younger participants. However, this pattern was differently expressed in the 2 age groups: In voxels where activity was increased during both SWM conditions compared with the control condition in older participants, the opposite effect was observed in younger participants. In addition, the within-condition scores differed between the older and younger age groups during both SWM conditions. The majority of robust voxels were located in posterior cingulate cortex, precuneus, bilateral angular gyrus, and medial prefrontal cortex (Fig. 2B, blue color, see Supplementary Table 5), and exhibited greater activity during both SWM conditions compared with the control condition in older participants; the opposite effect occurred in younger participants. Other voxels in right superior frontal and parietal cortices (Fig. 2B, red color, see Supplementary Table 5) exhibited greater activity during both SWM conditions relative to the control condition in younger participants, and the opposite effect (lower activity during SWM compared with the control condition) in older participants.

The results of the task PLS on the mean data showed that all participants recruited frontoparietal regions and disengaged some nodes of the DMN when performing the SWM task (LV1). In addition, younger participants further disengaged some nodes of the DMN while performing the SWM task (LV2). This latter effect was partly reversed in older adults, suggesting a reduced ability to suppress parts of the DMN or even a tendency to recruit them during the SWM task. To summarize the activation/deactivation patterns as identified by the task PLS, we computed a composite score such that the brain scores of the highand low-load conditions from the task PLS analysis were averaged for each participant, and then subtracted from the brain score of the control condition. For LV1, this composite score provides, for each participant, a measure of switch from the DMN to the frontostriatal regions when engaging in the SWM task. For LV2, the composite score provides a measure of deactivation (for positive numbers) or activation (for negative numbers) of the DMN when engaging in the SWM task.

Finally, we used univariate analyses to assess the relationship between the switch scores and the similar composite measure extracted from our initial behavioral PLS (brain scores averaged across load conditions) that summarize the relationship of $\mathrm{SD}_{\mathrm{BOLD}}$ to age, SWM accuracy, SWM RT, and D1 density in caudate and DLPFC. For each of 4 univariate models, the dependent variable was one of the 2 switch scores (LV1 or LV2), and the model included separate regressors for age, a brain score summarizing $\mathrm{SD}_{\mathrm{BOLD}}$ (behavioral PLS), and the interaction between the 2. We detected a significant effect relating the activation/deactivation of the DMN (LV2 task PLS, blue pattern in Fig. 2B) and the pattern of $\mathrm{SD}_{\mathrm{BOLD}}$ in neocortical regions that decreases with age and relates to better SWM accuracy (LV2 behavioral PLS, red pattern in Fig. 1B). In this model $\left(R^{2}=0.572\right.$, Fig. 3$)$, we found main effects of age $\left(F_{1,30}=4.6 ; P=0.04\right.$; partial $\left.\eta^{2}=0.13\right)$ and of neocortical BOLD variability $\left(F_{1,30}=8.66 ; P=0.006\right.$; partial $\left.\eta^{2}=0.22\right)$, as well as an interaction between these 2 variables $\left(F_{1,30}=8.7 ; P=0.006\right.$; partial $\eta^{2}=0.23$ ).

After excluding potential outliers using the Cook's distance (highlighted in Fig. 3; see Materials and Methods), the model still explained a substantial amount of variance $\left(R^{2}=0.539\right)$ and we still detected a main effect of neocortical variability

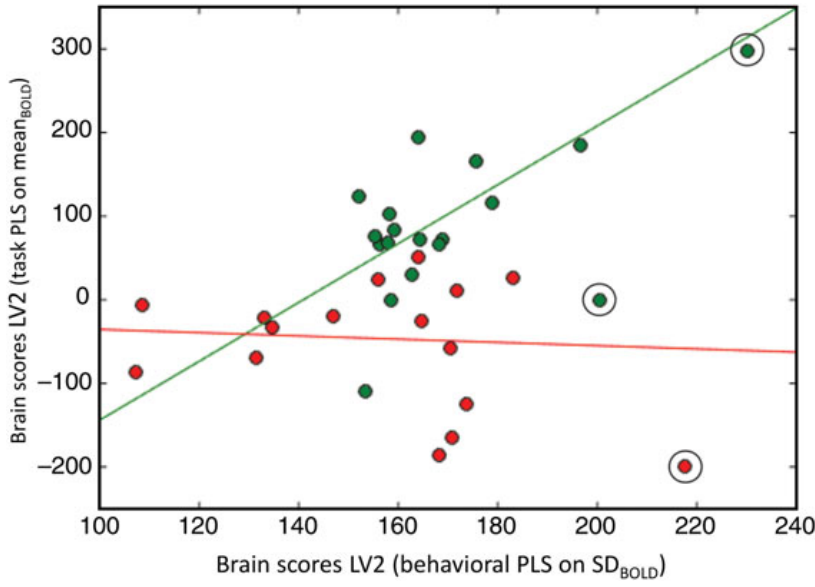

Figure 3. Relationship between BOLD variability and task-related deactivation of the DMN. Relationship between the brain scores from the task PLS (LV2; averaged high and low load-control) and the brain score from the behavioral PLS (LV2; averaged across load conditions). Older participants are depicted in red and younger participants are in green. Possible outliers as defined with a Cook's distance $>0.2$ are highlighted and excluded from the analysis. The extent of deactivation of the DMN is correlated with the amount of $\mathrm{SD}_{\mathrm{BOLD}}$ in neocortical regions in younger adults $\left(R^{2}=0.322, P=0.015 ; R^{2}=0.3, P=0.027\right.$ after removing the outliers), but not in older adults $\left(R^{2}=0.111, P=0.208 ; R^{2}=0.004, P=0.813\right.$ after removing the outlier)

$\left(F_{1,30}=5.14 ; P=0.032 ;\right.$ partial $\left.\eta^{2}=0.16\right)$ and an interaction between age and neocortical variability $\left(F_{1,30}=4.55 ; P=0.042\right.$; partial $\eta^{2}=$ $0.14)$; the main effect of age remained marginally significant $\left(F_{1,30}=2.78 ; P=0.107 ;\right.$ partial $\left.\eta^{2}=0.09\right)$. A stratified correlational analysis confirmed that, whereas in younger participants higher neocortical $\mathrm{SD}_{\mathrm{BOLD}}$ (which was functional) predicted more robust deactivation of the DMN during SWM $(r=0.55 ; P=0.027)$, neocortical $\mathrm{SD}_{\mathrm{BOLD}}$ did not predict the response of the DMN during SWM performance in older adults $(r=-0.07 ; P=0.813)$.

\section{Discussion}

We examined the relationship of D1 receptor densities in caudate and DLPFC to BOLD variability during SWM in the context of normal cognitive aging. Our analysis of spatial patterns reflecting associations of BOLD variability to age, SWM performance, and D1 receptor density in caudate and DLPFC detected 2 distinct patterns of voxels. Whereas both patterns reflected an association among BOLD variability, age, and SWM performance, only one reflected an association between BOLD variability and D1 receptor density. Specifically, BOLD variability was unrelated to D1 receptor densities in the majority of voxels in neocortex, where variability was higher in younger and more accurate participants. However, in some of these cortical voxels, mainly located in posterior cingulate cortex and medial prefrontal cortex, BOLD variability was associated with higher D1 receptor density. Despite this finding, the overall pattern suggests that dopaminergic receptor density may not be a key regulatory factor for level of BOLD variability in cortical regions where variability supports cognitive functioning (Garrett et al. 2010, 2011).

In contrast, $\mathrm{D} 1$ receptor densities were related to BOLD variability in a set of subcortical regions, including hippocampus, amygdala, thalamus, caudate nucleus, and midbrain. For these brain regions, neuronal variability was higher in older and slower participants. Importantly, the effects of D1 receptor density on BOLD variability survived when controlling for the effects of 
chronological age, motion, and brain atrophy. This pattern suggests that lower DA levels (Backman et al. 2000, 2006) contribute to elevated age-related subcortical BOLD variability.

These age-differential patterns regarding BOLD variability in neocortical versus subcortical regions are consistent with past research (Garrett et al. 2010, 2011; Samanez-Larkin et al. 2010). BOLD variability in large-scale neocortical patterns of voxels has been found to be higher in younger participants and related to better cognitive performance (Garrett et al. 2010, 2011). Similarly, studies using EEG and MEG show that neuronal variability increases during early development and maturation, and is related to enhanced cognitive performance (McIntosh et al. 2008; Lippe et al. 2009). As EEG and MEG are much more sensitive to cortical sources, it is likely that these studies also reflect neuronal variability at the cortical level. Collectively, these findings suggest that neuronal variability in neocortical regions reflects a healthy and well-functioning brain (Faisal et al. 2008; McIntosh et al. 2008, 2010; Garrett et al. 2010; 2011; Garrett, Kovacevic et al. 2013; Garrett, McIntosh et al. 2014; Garrett, Samanez-Larkin et al. 2013).

In contrast, in subcortical regions (e.g., striatum, thalamus) and in hippocampus, BOLD variability can be higher in older and poorer performers (Garrett et al. 2010, 2011; Samanez-Larkin et al. 2010). Interestingly, animal data indicate that impaired spatial memory in older rats is associated with higher trial-to-trial rearrangements of place fields of hippocampal neurons (Barnes et al. 1997; Wilson et al. 2004). Thus, evidence from diverse experimental sources supports the notion that neuronal variability in some brain regions may be dysfunctional and related to aging and cognitive decline. This raises the question why cortical and subcortical variability have differential effects on cognitive performance.

Our results provide novel information pertaining to this issue, suggesting that dopaminergic neurotransmission has a role in regulating BOLD variability in hippocampus and a set of subcortical regions including striatum. Higher BOLD variability in ventral striatum was observed when older participants performed a probabilistic decision-making task (Samanez-Larkin et al. 2010). In that study, the increase in signal variability mediated age-related increases in the number of suboptimal decisions (Samanez-Larkin et al. 2010). One possibility is that level of striatal BOLD variability is related to the integrity of the dopaminergic system. Indeed, decision-making performance in a probabilistic task and associated neuronal representations in ventral striatum are sensitive to dopaminergic drugs and related to the integrity of the nigrostriatal dopaminergic pathway as measured with Diffusion Tensor Imaging(Chowdhury et al. 2013). Collectively, these findings suggest that age-related decline in dopaminergic functions may cause increases in signal variability within identified subcortical regions that, in turn, result in altered neuronal representations and cognitive impairment.

Why DA modulates variability in subcortical, but not cortical, regions remains unknown. However, it is important to note that contemporary theories linking DA and neocortical activations refer to mean BOLD, whereas no previous report has studied the link between DA and BOLD variability. Given that BOLD variability and mean have proven to capture different aspects of the data (Garrett et al. 2010, 2011), it is plausible that DA have different effects on BOLD mean and BOLD variability in neocortical regions.

Recent theoretical work suggests that neuronal variability is required for the brain to flexibly adopt different network configurations (McIntosh et al. 2010; Deco et al. 2011). To test this hypothesis, we used a task PLS analysis to quantify patterns of voxels that show different mean BOLD responses for the different task conditions (McIntosh et al. 1996; Krishnan et al. 2011). We observed 2 distinct patterns. The first pattern was observed in both age groups and involved recruitment of a frontoparietalsubcortical set of regions and deactivation of the DMN during SWM performance. Activation in frontal and parietal regions is often observed in fMRI studies of working memory (Wager and Smith 2003; Salami et al. 2014). On the other hand, regions within the DMN are typically deactivated when participants engage in cognitive tasks (Raichle et al. 2001; Buckner et al. 2008). However, these task-related activations and deactivations were not related to levels of BOLD variability. Considering that this first pattern accounted for the majority of the cross-block covariance (>75\%), this result implies that, regardless of age, the brain responded similarly in response to the cognitive demands of the SWM task.

The second pattern revealed further deactivation of the DMN (posterior cingulate cortex, precuneus, bilateral angular gyrus, and medial prefrontal cortex) when performing the SWM task. However, this additional deactivation was only observed in younger participants. In contrast, older participants showed relative activation of these DMN regions when performing the SWM task. Furthermore, greater BOLD variability in neocortical regions, including posterior cingulate cortex (a recognized node of the DMN (Buckner et al. 2008)), was linked to greater deactivation of the DMN while performing the SWM task in younger participants. Although this relationship between neocortical variability and the efficiency in disengaging the DMN was only observed in younger adults, this finding provides a non-dopaminergic system-level mechanism by which neuronal variability is functional (Faisal et al. 2008; McIntosh et al. 2008, 2010; Garrett et al. 2010, 2011; Garrett, Kovacevic et al. 2013; Garrett, McIntosh et al. 2014; Garrett, Samanez-Larkin et al. 2013). The reason why BOLD variability in neocortical regions did not predict the response of the DMN during SWM in older adults is unknown. One possibility is that, as functional capacity decreases, older participants fail to inhibit the DMN when performing cognitively demanding tasks (Grady et al. 2010; Sambataro et al. 2010), and that this effect is independent from changes in BOLD variability.

This is the first study that addresses whether BOLD variability, a measure that is increasingly capturing attention as it relates to health and cognitive function, is linked to DA neurotransmission. In line with our hypotheses, the current study reveals higher BOLD variability in large-scale neocortical patterns of voxles in younger, more accurate performers, whereas BOLD variability in subcortical regions and hippocampus is higher in older and slower-performing adults. Our data also provide novel insights into the biological mechanisms that regulate levels of neuronal variability, and shows for the first time that level of BOLD variability in subcortical regions, including the hippocampus, is related to the integrity of the dopaminergic system. Furthermore, BOLD variability in neocortical regions is mostly unrelated to DA availability and we provide evidence suggesting a mechanism by which BOLD variability may be functional by showing that it supports younger adults' ability to disengage the DMN when performing a demanding cognitive task.

\section{Supplementary Material}

Supplementary material can be found at: http://www.cercor. oxfordjournals.org/.

\section{Funding}

This work was supported by grants from the Swedish Research Council (L.B.); Swedish Brain Power (L.B.); an Alexander von 
Humboldt Research Award (L.B).; a donation from the af Jochnick Foundation (L.B.); and the 2010 Wilhelm Gottfried Leibniz award of the German Research Foundation (U.L.).

\section{Notes}

Conflict of Interest: None declared.

\section{References}

Ashburner J. 2007. A fast diffeomorphic image registration algorithm. NeuroImage. 38:95-113.

Ashburner J, Friston KJ. 2005. Unified segmentation. NeuroImage. 26:839-851.

Bäckman L, Lindenberger U, Li SC, Nyberg L. 2010. Linking cognitive aging to alterations in dopaminergic neurotransmitter functioning: recent data and future avenues. Neurosci Biobehav Rev. 34:670-677.

Backman L, Karlsson S, Fischer H, Karlsson P, Brehmer Y, Rieckmann A, MacDonald SW, Farde L, Nyberg L. 2011. Dopamine $\mathrm{D}(1)$ receptors and age differences in brain activation during working memory. Neurobiol Aging. 32:1849-1856.

Backman L, Nyberg L, Lindenberger U, Li SC, Farde L. 2006. The correlative triad among aging, dopamine, and cognition: current status and future prospects. Neurosci Biobehav Rev. 30:791-807.

Backman L, Nyberg L, Soveri A, Johansson J, Andersson M, Dahlin E, Neely AS, Virta J, Laine M, Rinne JO. 2011. Effects of working-memory training on striatal dopamine release. Science. 333:718.

Barnes CA, Suster MS, Shen J, McNaughton BL. 1997. Multistability of cognitive maps in the hippocampus of old rats. Nature. 388:272-275

Beierholm U, Guitart-Masip M, Economides M, Chowdhury R, Duzel E, Dolan R, Dayan P. 2013. Dopamine modulates rewardrelated vigor. Neuropsychopharmacology. 38:1495-1503.

Bollen KA, Jackman RW. 1990. Regression diagnostics: an expository treatment of outliers and influential cases. In: Fox J, Long JS, editors. Modern methods of data analysis. Newbury Park, CA: Sage p 257-291.

Buckner RL, Andrews-Hanna JR, Schacter DL. 2008. The brain's default network: anatomy, function, and relevance to disease. Ann NY Acad Sci. 1124:1-38.

Chowdhury R, Guitart-Masip M, Lambert C, Dayan P, Huys Q Duzel E, Dolan RJ. 2013. Dopamine restores reward prediction errors in old age. Nat Neurosci. 16:648-653.

Cook RD. 1977. Detection of influential observation in linear regression. Technometrics. 19:15-18.

Cook RD. 1979. Influential observations in linear regression. J Am Stat Assoc. 365:169-174.

Cools R, D'Esposito M. 2011. Inverted-U-shaped dopamine actions on human working memory and cognitive control. Biol Psychiatry. 69:e113-e125.

Curtis CE, D'Esposito M. 2003. Persistent activity in the prefrontal cortex during working memory. Trends Cogn Sci. 7:415-423.

Deco G, Jirsa VK, McIntosh AR. 2011. Emerging concepts for the dynamical organization of resting-state activity in the brain. Nat Rev Neurosci. 12:43-56.

Faisal AA, Selen LP, Wolpert DM. 2008. Noise in the nervous system. Nat Rev Neurosci. 9:292-303.

Fischer H, Nyberg L, Karlsson S, Karlsson P, Brehmer Y, Rieckmann A, MacDonald SW, Farde L, Backman L. 2010. Simulating neurocognitive aging: effects of a dopaminergic antagonist on brain activity during working memory. Biol Psychiatry. 67:575-580.

Frey U, Morris RG. 1997. Synaptic tagging and long-term potentiation. Nature. 385:533-536.

Garrett DD, Kovacevic N, McIntosh AR, Grady CL. 2010. Blood oxygen level-dependent signal variability is more than just noise. J Neurosci. 30:4914-4921.

Garrett DD, Kovacevic N, McIntosh AR, Grady CL. 2011. The importance of being variable. J Neurosci. 31:4496-4503.

Garrett DD, Kovacevic N, McIntosh AR, Grady CL. 2013. The modulation of BOLD variability between cognitive states varies by age and processing speed. Cereb Cortex. 23:684-693.

Garrett DD, McIntosh AR, Grady CL. 2014. Brain signal variability is parametrically modifiable. Cereb Cortex. 24:2931-2940.

Garrett DD, Samanez-Larkin GR, MacDonald SW, Lindenberger U, McIntosh AR, Grady CL. 2013. Moment-to-moment brain signal variability: a next frontier in human brain mapping? Neurosci Biobehav Rev. 37:610-624.

Grady CL, Protzner AB, Kovacevic N, Strother SC, Afshin-Pour B, Wojtowicz M, Anderson JA, Churchill N, McIntosh AR. 2010. A multivariate analysis of age-related differences in default mode and task-positive networks across multiple cognitive domains. Cereb Cortex. 20:1432-1447.

Guitart-Masip M, Chowdhury R, Sharot T, Dayan P, Duzel E, Dolan RJ. 2012. Action controls dopaminergic enhancement of reward representations. Proc Natl Acad Sci U S A. 109:7511-7516.

Hall H, Farde L, Sedvall G. 1988. Human dopamine receptor subtypes-in vitro binding analysis using 3H-SCH 23390 and 3Hraclopride. J Neural Transm. 73:7-21.

Innis RB, Cunningham VJ, Delforge J, Fujita M, Gjedde A, Gunn RN, Holden J, Houle S, Huang SC, Ichise M, et al. 2007. Consensus nomenclature for in vivo imaging of reversibly binding radioligands. J Cereb Blood Flow Metab. 27:1533-1539.

Krishnan A, Williams LJ, McIntosh AR, Abdi H. 2011. Partial Least Squares (PLS) methods for neuroimaging: a tutorial and review. NeuroImage. 56:455-475.

Landau SM, Lal R, O'Neil JP, Baker S, Jagust WJ. 2009. Striatal dopamine and working memory. Cereb Cortex. 19:445-454.

Laukka EJ, Lovden M, Herlitz A, Karlsson S, Ferencz B, Pantzar A, Keller L, Graff C, Fratiglioni L, Backman L. 2013. Genetic effects on old-age cognitive functioning: a population-based study. Psychol Aging. 28:262-274.

Li SC, Lindenberger U, Sikstrom S. 2001. Aging cognition: from neuromodulation to representation. Trends Cogn Sci. 5:479-486.

Lippe S, Kovacevic N, McIntosh AR. 2009. Differential maturation of brain signal complexity in the human auditory and visual system. Front Hum Neurosci. 3:48.

Lisman JE, Grace AA. 2005. The hippocampal-VTA loop: controlling the entry of information into long-term memory. Neuron. 46:703-713.

MacDonald SW, Karlsson S, Rieckmann A, Nyberg L, Backman L. 2012. Aging-related increases in behavioral variability: relations to losses of dopamine D1 receptors. J Neurosci. 32:8186-8191.

McDonnell MD, Ward LM. 2011. The benefits of noise in neural systems: bridging theory and experiment. Nat Rev Neurosci. 12:415-426.

McIntosh AR, Bookstein FL, Haxby JV, Grady CL. 1996. Spatial pattern analysis of functional brain images using partial least squares. NeuroImage. 3:143-157.

McIntosh AR, Kovacevic N, Itier RJ. 2008. Increased brain signal variability accompanies lower behavioral variability in development. PLoS Comput Biol. 4:e1000106. 
McIntosh AR, Kovacevic N, Lippe S, Garrett D, Grady C, Jirsa V. 2010. The development of a noisy brain. Arch Ital Biol. 148:323-337.

Meltzer CC, Leal JP, Mayberg HS, Wagner HN Jr, Frost JJ. 1990. Correction of PET data for partial volume effects in human cerebral cortex by MR imaging. J Comput Assist Tomogr. 14:561-570.

Niv Y, Daw ND, Joel D, Dayan P. 2007. Tonic dopamine: opportunity costs and the control of response vigor. Psychopharmacology. 191:507-520.

Nyberg L, Andersson M, Forsgren L, Jakobsson-Mo S, Larsson A, Marklund P, Nilsson LG, Riklund K, Backman L. 2009. Striatal dopamine $\mathrm{D} 2$ binding is related to frontal BOLD response during updating of long-term memory representations. NeuroImage. 46:1194-1199.

Raichle ME, MacLeod AM, Snyder AZ, Powers WJ, Gusnard DA, Shulman GL. 2001. A default mode of brain function. Proc Natl Acad Sci U S A. 98:676-682.

Rieckmann A, Karlsson S, Fischer H, Backman L. 2011. Caudate dopamine D1 receptor density is associated with individual differences in frontoparietal connectivity during working memory. J Neurosci. 31:14284-14290.

Roland PE, Graufelds CJ, J WH, Ingelman L, Andersson M, Ledberg A, Pedersen J, Akerman S, Dabringhaus A, Zilles K.
1994. Human brain atlas: For high-resolution functional and anatomical mapping. Hum Brain Mapp. 1:173-184.

Salami A, Rieckmann A, Fischer H, Backman L. 2014. A multivariate analysis of age-related differences in functional networks supporting conflict resolution. NeuroImage. 86:150-163.

Salamone JD, Correa M, Farrar A, Mingote SM. 2007. Effort-related functions of nucleus accumbens dopamine and associated forebrain circuits. Psychopharmacology. 191:461-482.

Samanez-Larkin GR, Kuhnen CM, Yoo DJ, Knutson B. 2010. Variability in nucleus accumbens activity mediates age-related suboptimal financial risk taking. J Neurosci. 30:1426-1434.

Sambataro F, Murty VP, Callicott JH, Tan HY, Das S, Weinberger DR, Mattay VS. 2010. Age-related alterations in default mode network: impact on working memory performance. Neurobiol Aging. 31:839-852.

Schultz W, Dayan P, Montague PR. 1997. A neural substrate of prediction and reward. Science. 275:1593-1599.

Wager TD, Smith EE. 2003. Neuroimaging studies of working memory: a meta-analysis. Cogn Affect Behav Neurosci. 3:255-274.

Wilson IA, Ikonen S, Gureviciene I, McMahan RW, Gallagher M, Eichenbaum H, Tanila H. 2004. Cognitive aging and the hippocampus: how old rats represent new environments. J Neurosci. 24:3870-3878. 